\title{
Synthesis of Carbon Microspheres from Saccharides Based on Hydrothermal Process
}

\author{
Shunjian $\mathrm{Xu} *$, Yufeng Luo, Wei Zhong, Zonghu \\ Xiao, Yongping Luo, Hui Ou and Huanwen $\mathrm{Wu}$ \\ Xinyu Institute of New Energy \\ Xinyu University \\ Xinyu, P. R. China \\ e-mail: xushunjian@126.com
}

\author{
Feng Ye \\ School of Materials Science and Engineering \\ Chang'an University \\ Xi'an, P. R. China
}

\begin{abstract}
Three types of saccharides including glucose, fructose and starch were used as the carbon precursors to synthesize carbon microspheres (CMSs) through hydrothermal process and subsequently pyrolysis, with emphasis on investigating the effect of the types of saccharides on the particle size and morphology of the obtained CMSs. The scanning electron microscopy (SEM) shows that the CMSs derived from each saccharide are in perfect spherical morphologies whose surfaces are very smooth without cracks. The article size analysis based on the SEM images reveals the particle sizes of the CMSs are controlled by altering the types of saccharides. The mean particle sizes of the CMSs derived from glucose, starch and fructose are 244,528 and $1060 \mathrm{~nm}$, respectively. The $\mathrm{X}$-ray diffraction displays that the influence of the types of saccharides on the crystal structure of the CMSs can be ignorable. The amorphous carbon phases are formed within all CMSs. The pyrolysis process has no effect on the morphological type of the CMSs but results in particle size contraction, surface smooth and crystal structure transformation. The size shrinkages are around $13.5 \%$.
\end{abstract}

Keywords-saccharide; carbon microspheres; hydrothermal process; pyrolysis

\section{INTRODUCTION}

In the past few years, carbon microspheres (CMSs) have attracted a great deal of research interest because of their unique performances in double-layer capacitors, catalyst supports, adsorbents, lithium-ion secondary battery and templates for fabricating core-shell [1-3]. Since the application values of the CMSs significantly depend on the morphology and particle size of the particles, different techniques have been developed to synthesize and control the CMSs, such as chemical vapor deposition, reduction of carbides with metal catalysis, high-temperature pyrolysis and hydrothermal process $[4,5]$. Among them, the hydrothermal process is unique one owning to its simple operational and mild reaction condition requirements. In the hydrothermal process, some saccharides, such as glucose and sugar, are used as the carbon precursors for preparing the CMSs [6-9]. Mi et al [7] have employed glucose as carbon precursor to synthesize the CMSs with a regular and perfect shape, high yields and narrow size distribution (from 1 to $2 \mu \mathrm{m}$ ). Li et al [8] have also used glucose as carbon precursor to prepare the
CMSs, with emphasis on investigating the effects of the hydrothermal parameters, including the concentration of glucose, reaction temperature, duration, and the second hydrothermal treatment. Wang et al [9] have selected sugar as carbon precursor to fabricate CMSs with perfect spherical morphology, and investigated the effect of the concentration of sugar solution on the size and morphology of the CMSs. However, there are a few reports to investigate the effect of the types of saccharides on the particle size and morphology of the CMSs.

In this study, three types of saccharides were individually introduced into the hydrothermal process as the carbon precursors to investigate the differences in the particle size, morphology and crystal structure of the obtained CMSs. The saccharides referred to glucose, fructose and starch.

\section{EXPERIMENTAL}

\section{A. Materials}

The glucose in the form of $\alpha$-D-(+)-Glucose (99\%) and the fructose in the form of D-(-)-Fructose (99\%) were obtained from J\&K Scientific Co. Ltd. The starch (Pharmaceutical grade, water-solubility) was obtained from Aladdin Industrial Inc. The above saccharides were used without further purification. The Molecular structure diagrams of the saccharides are shown in Figure 1.

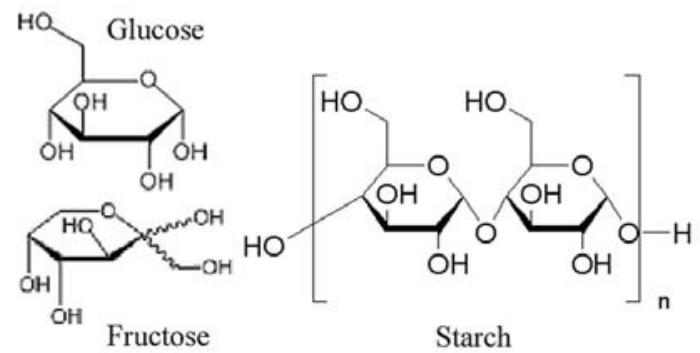

Figure 1. Molecular structure diagrams of three saccharides.

\section{B. Synthesis of CMSs}

In the hydrothermal process, $5 \mathrm{~g}$ of saccharide was first dissolved in $40 \mathrm{~mL}$ of deionized water and magnetically 
stirred for $2 \mathrm{~h}$. The solution was then sealed in a $50 \mathrm{~mL}$ stainless-steel autoclave, which was put into an oven and heated at $190{ }^{\circ} \mathrm{C}$ for $6 \mathrm{~h}$. After the autoclave was cooled to room temperature, the precipitates were collected and washed with acetone, distilled water and ethanol, and dried in a vacuum at $80^{\circ} \mathrm{C}$ for $6 \mathrm{~h}$ to obtain the powder products. In the pyrolysis process, the powder products were heated at $600{ }^{\circ} \mathrm{C}$ for $1 \mathrm{~h}$ under a flowing $\mathrm{N}_{2}$ atmosphere to synthesize the CMSs. The used saccharides were involved in glucose, fructose and starch. The processing steps for the synthesis of the CMSs are shown in Figure 2.

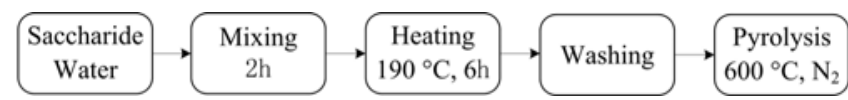

Figure 2. Processing steps for the synthesis of the CMSs.

\section{Characterizations}

The morphologies of the samples were characterized by a scanning electron microscopy (SEM; ¿IGMA, Zeiss, Germany). Particle size parameters and particle size distributions of the samples were estimated by Nano Measurer based on the SEM images. The crystal structures of the samples were identified by an $\mathrm{X}$-ray diffraction (XRD; D8 ADVANCE, Bruker, Germany) using CuK $\alpha$ radiation. The scanning step was $0.02^{\circ}$, and the scanning area was $15-75^{\circ}$.

\section{Results AND Discussion}

\section{A. Morphologies of CMSs from Different Saccharides}

The typical morphologies of the CMSs prepared by hydrothermal process and subsequently pyrolysis are shown in Figure 3. It is obvious that the final pyrolyzed products derived from each saccharide consist of a large quantity of CMSs. All the CMSs are in perfect spherical morphologies whose surfaces are very smooth without cracks. More information can be obtained from Figure 3 that the particle sizes of the CMSs from various saccharides show significant differences. The particle size of the starch derived CMSs is smaller than that of the fructose derived CMSs, but bigger than that of the glucose derived CMSs.

\section{B. Particle Size of CMSs from Different Saccharides}

The particle size parameters of the CMSs are revealed in Table I. The mean particle sizes of the CMSs prepared from starch, fructose and glucose are 528, 1060 and $244 \mathrm{~nm}$, respectively. Figure 4 displays the particle size distributions of the CMSs. When glucose and fructose are used as carbon precursors, the particle size distributions are narrow. Main particles in the CMSs from glucose and fructose distribute in the ranges of $150 \sim 300 \mathrm{~nm}$ and $900 \sim 1200 \mathrm{~nm}$, respectively. In contrast, when starch is employed as carbon precursor, the CMSs have broad range of particle size distribution. Main particles range from 300 to $800 \mathrm{~nm}$. The analyses of Figure 3 , Figure 4 and Table I suggest that the particle sizes of the CMSs based on hydrothermal process can be controlled by altering the carbon precursors.
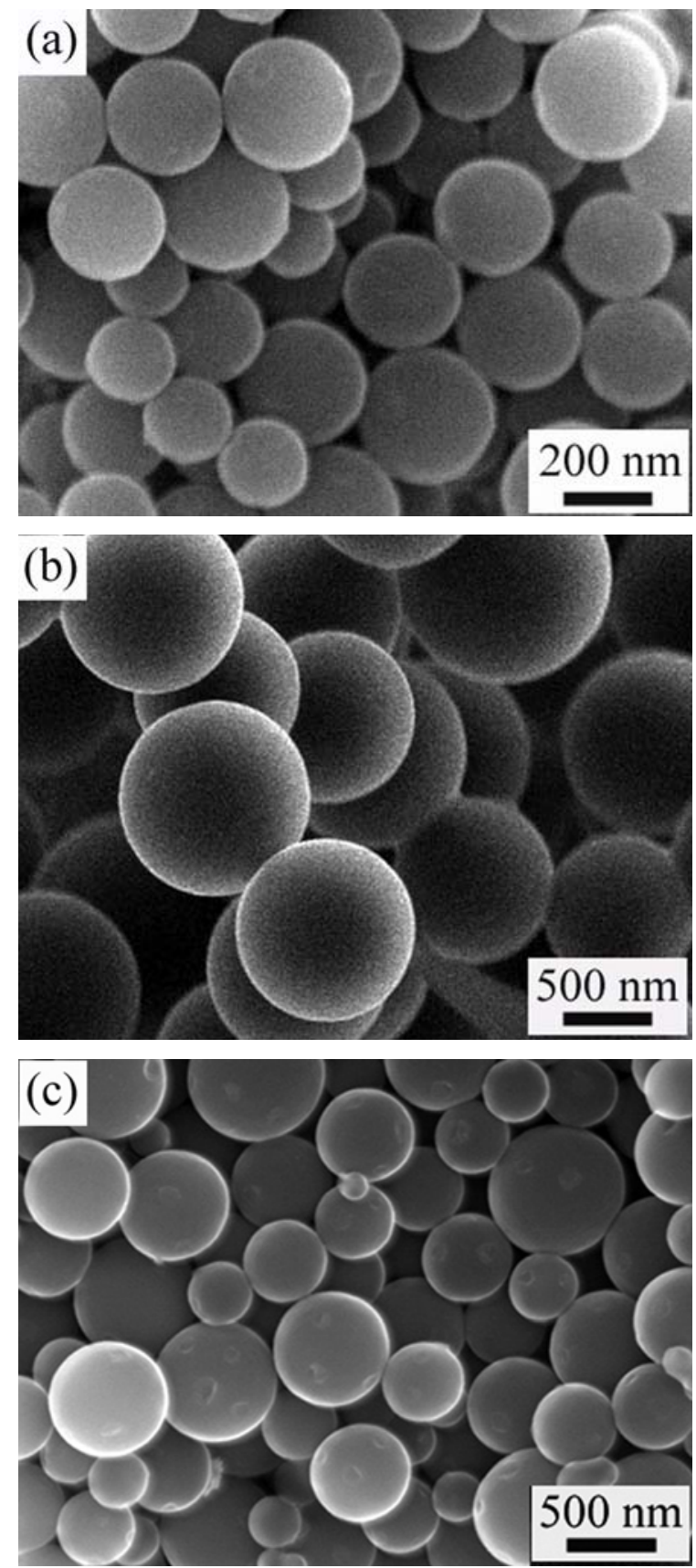

Figure 3. SEM morphologies of CMSs derived from various saccharides: (a) Glucose, (b) Fructose and (c) Starch.

table I. Particle Size Parameters of CMSs Derived From VARIOUS SACCHARIDES.

\begin{tabular}{|c|c|c|c|}
\hline Saccharides & $\begin{array}{c}\text { Mean } \\
\text { particle size } \\
\text { /nm }\end{array}$ & $\begin{array}{c}\text { Maximum } \\
\text { particle size } \\
\text { /nm }\end{array}$ & $\begin{array}{c}\text { Minimum } \\
\text { particle } \\
\text { size } / \mathbf{n m}\end{array}$ \\
\hline Glucose & 244 & 314.71 & 156.76 \\
\hline Fructose & 1060 & 1249.74 & 680.05 \\
\hline Starch & 528 & 1005.99 & 196.40 \\
\hline
\end{tabular}




\section{Crystal Structure of CMSs from Different Saccharides}

The XRD patterns of the CMSs from various saccharides are demonstrated in Figure 5. Two broad peaks of (002) and (100) are observed in each XRD pattern. Both peaks can be indexed to a hexagonal graphite lattice. The broadening of the peaks means the presence of an amorphous carbon phase within the CMSs [10]. Noted that the position and intensity of the (002) and (100) peaks among the three CMSs are almost identical, indicating that the influence of the saccharide types on the crystal structure of the CMSs can be ignorable.

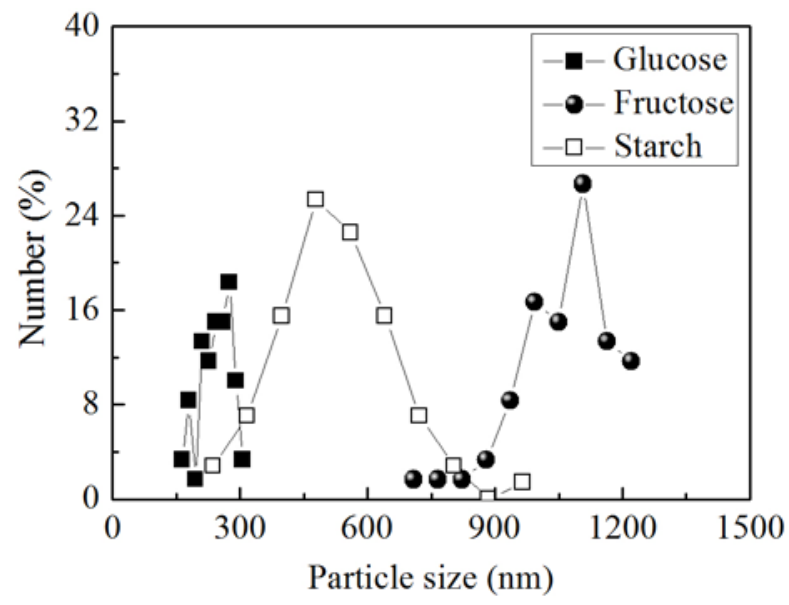

Figure 4. Particle size distributions of MCSs derived from various saccharides.

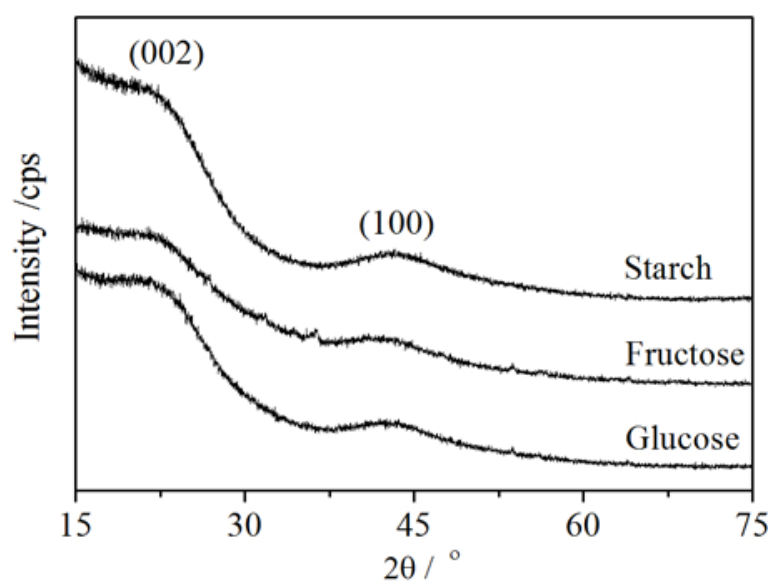

Figure 5. XRD patterns of the MCSs derived from various saccharides.

\section{Effect of Pyrolysis Process on the CMSs}

To investigate the effect of the pyrolysis process on the CMSs, the characterizations of the glucose derived powder products formed during hydrothermal process (before pyrolysis) have been identified, as shown in Figure 6. The SEM morphology in Figure 6(a) reveals that perfect spherical shape can be observed in the products, which means that the morphological type of the CMSs is inherited from that of the microspheres formed during hydrothermal process. Noted that the surface of the microspheres before pyrolysis is slightly coarser than that of the CMSs. The particle size distribution in Figure 6(b) displays that main microspheres before pyrolysis distribute in the range from 250 to $350 \mathrm{~nm}$. Moreover, the mean particle size of the microspheres before pyrolysis is $277 \mathrm{~nm}$, which is slightly bigger than that of the CMSs. The above results suggest that the size of all microspheres contracts during pyrolysis, and the size shrinkages are about $13.5 \%$. The XRD pattern in Figure 6(c) shows that the amorphous carbon phase is unformed within the microspheres before pyrolysis since the (100) peak is not occurred.
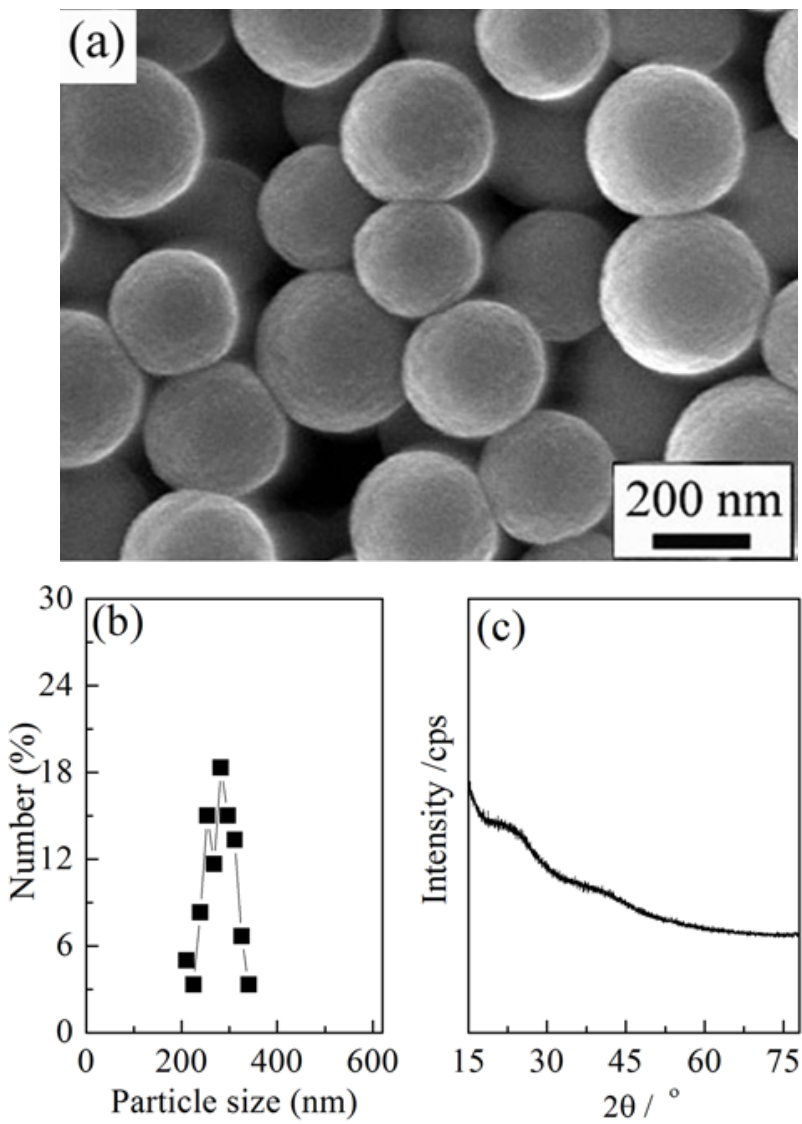

Figure 6. Characterizations of glucose derived powder products before pyrolysis: (a) SEM morphology, (b) Particle size distribution and (c) XRD pattern.

\section{CONClusions}

The CMSs are synthesized employing three saccharides as carbon precursors by hydrothermal process and subsequently pyrolysis. The CMSs derived from all saccharides are in perfect spherical morphologies whose surfaces are very smooth without cracks. The particle size of the CMSs can be controlled by altering the saccharides. When the glucose and fructose are used as the carbon precursors, the particle size distributions are narrow, and main particles distribute in the ranges of $150 \sim 300 \mathrm{~nm}$ and $900 \sim 1200 \mathrm{~nm}$, respectively. When the starch is employed as the carbon precursor, the particle size distribution of CMSs is broad, and main particles range from 300 to $800 \mathrm{~nm}$. The 
mean particle sizes of the CMSs derived from glucose, fructose and starch are 244, 1060 and $528 \mathrm{~nm}$, respectively. The pyrolysis process has no effect on the morphological type of the CMSs but results in the contraction of particle size with shrinkage of about $13.5 \%$, the smooth of surface and the transformation of the crystal structure into the amorphous carbon phases. Moreover, the influence of the saccharides on the crystal structure of the CMSs can be ignorable.

\section{ACKNOWLEDGMENT}

This work was financially supported by National Natural Science Foundation of China (51162025, 51164033), Training Programme Foundation for Young Scientist of Jiangxi, China (20133BCB23035) and Educational Commission of Jiangxi, China (KJLD13100).

\section{REFERENCES}

[1] M. Wu, J. Liu, M. H. Tan, Z. T. Li, W. T. Wu, Y. P. Li, H. P. Wang, and J. Y. Zheng, et al., "Facile hydrothermal synthesis of $\mathrm{SnO}_{2} / \mathrm{C}$ microspheres and double layered core-shell $\mathrm{SnO}_{2}$ microspheres as anode materials for Li-ion secondary batteries," RSC Advances, Vol. 4, pp. 25189-25194, May 2014.

[2] S. Du, L. Wang, X. Fu, M. Chen, and C. Wang, "Hierarchical porous carbon microspheres derived from porous starch for use in high-rate electrochemical double-layer capacitors," Bioresource Technol., Vol. 139, pp. 406-409, April 2013.
[3] H. Xiong, M. Moyo , M. A. Motchelaho, Z. N. Tetana, S. M. A. Dube, and L. L. Jewell, et al., "Fischer-Tropsch synthesis: Iron catalysts supported on N-doped carbon spheres prepared by chemical vapor deposition and hydrothermal approaches," J. Catal., Vol. 311, pp. 80-87, March 2014.

[4] S. Zhao, X. Li, C. Wang, and M. Chen, "Preparation of bowl-like and eggshell-like hollow carbon microspheres from potato starch," Mater. Lett., Vol. 70, pp. 54-56, March 2012.

[5] G. Pari, S. Darmawan, and B. Prihandoko, "Porous carbon spheres from hydrothermal carbonization and $\mathrm{KOH}$ activation on cassava and tapioca flour raw material," Procedia Environ. Sci., Vol. 20, pp. 342 351, 2014.

[6] S. Ratchahat, N. Viriya-empikul, K. Faungnawakij, T. Charinpanitkull, and A. Soottitantawat, "Synthesis of carbon microspheres from starch by hydrothermal process," Sci. J. UBU, Vol. 1, pp. 40-45, December 2010.

[7] Y. Mi, W. Hu, Y. Dan, and Y. Liu, "Synthesis of carbon microspheres by a glucose hydrothermal method," Mater. Lett., Vol. 62, pp. 1194-1196, March 2008.

[8] M. Li, W. Li, and S. Liu, "Hydrothermal synthesis, characterization, and $\mathrm{KOH}$ activation of carbon spheres from glucose," Carbohyd Res., Vol. 346, pp. 999-1004, June 2011.

[9] Q. Wang, H. Li, L. Q. Chen, and X. J. Huang, "Monodispersed hard carbon spherules with uniform nanopores," Carbon, Vol. 39, pp. 2211-2214, November 2001.

[10] S. J Xu, Y. F Luo, and W. Zhong, "Investigation of catalytic activity of glassy carbon with controlled crystallinity for counter electrode in dye-sensitized solar cells," Sol. Energ., Vol. 85, pp. 2826-2832, November 2011. 